\title{
Axiomatic Local Metric Derivatives for Low-Level Fractionality with Mittag-Leffler Eigenfunctions
}

\author{
J. Weberszpil ${ }^{*}$ and J. A. Helayël-Neto ${ }^{+}$ \\ *Programa de Pós Gradiuação em Modelagem Matemática e Computacional-PPGMMC, Universidade Federal \\ Rural do Rio de Janeiro, UFRRJ-IM/DTL \\ Av. Governador Roberto Silveira s/n- Nova Iguaçú, Rio de Janeiro, Brasil, 695014. \\ josewebe@gmail.com \\ ${ }^{+}$Centro Brasileiro de Pesquisas Físicas-CBPF \\ Rua Dr Xavier Sigaud 150, 22290-180, Rio de Janeiro RJ Brasil. \\ helayel@cbpf.br
}

\begin{abstract}
In this contribution, we build up an axiomatic local metric derivative that exhibits Mittag-Leffler function as an eigenfunction and is valid for low-level fractionality, whenever the order parameter is close to 1 . This version of deformed (or metric) derivative may be a possible alternative to the versions worked out by Jumarie and the so-called local fractional derivative also based on Jumarie's approach. With rules similar to the classical ones, but with a systematic axiomatic basis in the limit pointed out here, we present our results and some comments on the limits of validity for the controversial formalism found in the literature of the area.
\end{abstract}

\section{Indexing terms/Keywords}

Deformed Derivatives, Metric Derivatives, Fractal Continuum, Mittag-Leffler Function, Eigenfunction, Low Level Fractionality

\section{Academic Discipline And Sub-Disciplines}

Physics/Mathematics.

\section{SUBJECT CLASSIFICATION}

Mathematical Methos in Physics.

\section{TYPE (METHOD/APPROACH)}

Theoretical: Mathematical Methos in Physics- Deformed or metric derivatives.

\section{INTRODUCTION}

In recent works, we have developed connections and a variational formalism for deformed or metric derivatives, considering the relevant space-time/ phase space as fractal or multifractal $[1,2]$.

The use of deformed-operators was justified based on our proposition that there exists an intimate relationship between dissipation, coarse-grained media and the some limit scale of energy for the interactions. Concepts and connections like open systems, quasi-particles, energy scale and the change of the geometry of space-time at its topology level, nonconservative systems, noninteger dimensions of space-time connected to a coarse-grained medium, all of this were discussed. With this perspective, we argued that deformed or we should say, metric derivatives, similarly to the Fractional Calculus (FC), could allows us to describe and emulate certain dynamics without explicit many-body, dissipation or geometrical terms in the dynamical governing equations. Also, we emphasized that the paradigm we adopt were different from the standard approach in the generalized statistical mechanics context $[\underline{3}, \underline{4}, \underline{5}$, where the modification of entropy definition leads to the modification of algebra and consequently the derivative concept [1, 2]. This was structured by mapping to a continuous fractal space $[\underline{6}, \underline{7}, \underline{8}]$ which leads naturally to the necessity of modifications in the derivatives, that we called deformed or better than, metric derivatives [1, 2]. The modifications of derivatives, accordingly with the metric, bring to a change in the algebra involved, which in turn may conduct to a generalized statistical mechanics with some adequate definition of entropy.

In this communication, we develop, by means of an axiomatic approach, a local metric derivative that has the MittagLeffler function as an eigenfunction and for which deformed Leibniz' and the chain rule hold, similarly to the standard calculus, but whenever a low-level of fractionality is considered. The deformed operators here are local.

Our main goal here is to show that it is possible to build up a local formalism that meets certain similarities to those required by a version of fractional calculus attributed to Jumarie $[9, \underline{10}, 11]$, in such a way that reconciles the relevant axioms of the approach outlined here and, additionally, it is an approximation valid within a low fracionality limit. In this way, we try to make clear, in short, that existing studies in the literature that apply the Modified Riemann Liouville (MRL) derivative definition [9], and there are so many, are in fact local and behave as an approximations but, provided that the order of the derivative is very close to one. That is, being local and considered as approximations. 
Our paper is outlined as follows: In Section 2, some motivations are collected; in Section 3, we cast some mathematical aspects; in Section 4, we build up an axiomatic local metric derivative. We finally present our Conclusions and Outlook in Section 5

\section{THE MOTIVATION}

Some initial claims here coincide with our work of Refs. [1, 2]. The local differential equation $\frac{d y}{d x}=y^{q}$, yields the solution given by the q-exponential, $y=e^{q}[\underline{3}, \underline{4}, \underline{5}]$.

The differential equation, with the local Hausdorff derivative proposed in Ref. [12], reads:

$$
\frac{d^{H} y}{d x^{\alpha}}=y
$$

and leads to the stretched exponential solution $y=e^{x^{\alpha}}$.

But, in terms of nonlocal fractional calculus, the fractional differential equation

$$
\frac{d^{\alpha} y}{d x^{\alpha}}=y
$$

with the Caputo fractional derivative approach, yields the solution in terms of the Mittag-Leffler function $y=E_{\alpha}\left(x^{\alpha}\right)$.

The Mittag-Leffler function is of extreme importance to describe the dynamics of complex systems. It involves a generalization of exponential function and several trigonometric ands hyperbolic functions.

The problem with FC is that Leibniz rule and the chain rule of traditional calculus are violated in their standard forms, which makes it difficult to apply the mathematical tool in areas such as field and particle physics. Other problems are the fact that the derivative of a constant is not zero in the Riemann-Liouville approach and that there are strong restrictions for the class of smooth functions in the Caputos's approach.

Some authors have been attempting to come over those difficulties by redefining some expressions of fractional integral and derivatives [9], arguing that by this way the basic rules of usual differential and integral calculus would be nonviolated. Unfortunately several mistakes here pointed out for these attempts [13, 14, $\underline{15}, \underline{16}$, 17]. Recently, we have suggested that the mentioned approaches were in fact based on local operators [1].

In this sense, there emerges a question: is it possible to have a local deformed operator whose eigenfunctions could be the Mittag-Leffler function and preserves the non-violated form of Leibniz rule, with some easy form for chain rule, even if the rules were considered as approximations? This is our actual goal here. To propose some local deformed or metric derivatives that should satisfy those requests, but being local and an approximation for low level of fractionality (order parameter close to 1).

An important point to consider is a possible application for the approach proposed here. These considerations shall be tackled in our Conclusions.

\section{A BRIEF GLANCE AT MATHEMATICAL ASPECTS}

Here, in this Section, we provide some brief information to remember the main forms of deformed or metric derivative. The readers may see ref. $[1, \underline{2}, \underline{6}]$ for more details.

\section{Hausdorff Derivative}

By employing the local fractional differential operators in connection with the Hausdorff derivative [12], we can write that:

$$
\frac{d^{H}}{d x^{\varsigma}} f(x)=\lim _{x \rightarrow x^{\prime}} \frac{f\left(x^{\prime}\right)-f(x)}{\left(x^{\prime}\right)^{\varsigma}-x^{\varsigma}}=\left(\frac{x}{l_{0}}+1\right)^{1-\varsigma} \frac{d}{d x} f=\frac{l_{0}^{\varsigma^{-1}}}{c_{1}} \frac{d}{d x} f=\frac{d}{d^{\varsigma} x} f,
$$

where $I_{0}$ is the lower cutoff along the Cartesian $x$-axis and the scaling exponent, $\zeta$, characterizes the density of states along the direction of the normal to the intersection of the fractal continuum with the plane, as defined in the work [6].

\section{Conformable derivative}

This kind of deformed derivative has been proposed by the authors of Ref. [18]; it preserves classical operational properties and is given by

$$
T_{\alpha} f(t)=\lim _{\varepsilon \rightarrow 0} \frac{f\left(t+\varepsilon t^{1-\alpha}\right)-f(t)}{\varepsilon} .
$$

If the function is differentiable in a classical sense, the definition above yields 


$$
T_{\alpha} f(t)=t^{1-\alpha} \frac{d f(t)}{d t}
$$

Performing the change of variable $t \rightarrow 1+\frac{x}{l_{0}}$, we have shown that eq.(므) is nothing but the Hausdorff derivative up to a constant and valid for differentiable functions [1].

Another similar definition of local deformed derivative with classical properties is the one used in Ref. [19]:

Let $f:[0, \infty) \rightarrow \mathbb{R}$ and $t>0$. Then, the local deformed derivative-Katugampola- of $f$ of order $\alpha$ is defined by,

$$
D^{\alpha} f(t)=\lim _{\varepsilon \rightarrow 0} \frac{f\left(t e^{\varepsilon t^{-\alpha}}\right)-f(t)}{\varepsilon} .
$$

for $t>0, \alpha \in(0,1)$. If $f$ is $\alpha$-differentiable in some $(0, a), a>0$ and $\lim _{t \rightarrow 0^{+}} D^{\alpha} f(t)$ exists, then define

$$
D^{\alpha} f(0)=\lim _{t \rightarrow 0^{+}} D^{\alpha} f(t)
$$

\section{q-derivative in a nonextensive context}

With the generalized nonaddictive $q$-entropy as the main motivation, the $q$-derivative sets up a deformed algebra and takes into account that the $q$-exponential is eigenfunction of $D_{(q)}[20$. Borges proposed the operator for $q$-derivative as given below:

$$
D_{(q)} f(t)=\lim _{y \rightarrow x} \frac{f(x)-f(y)}{x \boldsymbol{\Theta}_{q} y}=[1+(1-q) x] \frac{d f(x)}{d x} .
$$

Here, $\boldsymbol{\theta}_{q}$ is the deformed difference operator, $x \boldsymbol{\Theta}_{q} y=\frac{x-y}{[1+(1-q) y]},(y \neq 1 /(q-1))$.

Recently, we have shown [1] that the main parameters can be connected as:

$$
1-q=\frac{(1-\varsigma)}{l_{0}}
$$

So, we conclude that the deformed $q$-derivative is the first-order expansion of the Hausdorff derivative and that there is a strong connection between these formalisms by means of a fractal metric.

For further more details, the reader may consult the refs. [18, 19].

\section{The Leibiniz' and the chain rule hold for metric derivative}

As the reader can readily check, for the metric derivatives the Leibniz rule holds $D^{\alpha}(f g)=g D^{\alpha} f+f D^{\alpha} g$; and similarly, for q-derivative: $D_{q}(f g)=g D_{q} f+f D_{q} g$. For composed functions, the chain rule holds true as well:

$$
\begin{aligned}
& D^{\alpha}(f \circ g)(x)=\frac{d f(g(x))}{d g} D^{\alpha} g(x), \\
& D_{q}(f \circ g)(x)=\frac{d f(g(x))}{d g} D_{q} g(x) .
\end{aligned}
$$

\section{AXIOMATIC LOCAL METRIC DERIVATIVE}

In this Section, we pursue the investigation of a version of metric derivative that has the Mittag-Leffler functions as eigenfunctions. This is due to the fact that the appearance of Mittag-Leffler function is very present in the description of dynamics for some complex systems like soil, porous media or some viscous systems.

- Axiom 1. Linearity

$D^{\alpha}\left[c_{1} f(x)+c_{2} g(x)\right]=c_{1} D^{\alpha} f(x)+c_{2} D^{\alpha} g(x)$.

- Axiom 2. Leibniz Rule

$D^{\alpha}(f g)=\left(D^{\alpha} f\right) g+f\left(D^{\alpha} g\right)$. 


\section{- Axiom 3. Derivative of a Power Function}

$$
D^{\alpha}(t-a)^{v}=\frac{\Gamma(1+v)}{\Gamma(1+v-\alpha)}(t-a)^{v-\alpha},(\alpha, v, x>0),
$$

\section{- Axiom 4. Low-Level Fractionality}

Here, we consider $\alpha$ very close to 1 . This is what we call low-level fractionality.

Clearly, $D^{\alpha} 1=0$, since the Leibniz rule is assumed.

We consider axiom 4 to give consistency to the the simultaneous validity of the axioms 2 and 3 . So, all counter-examples for incompatibility are actually inappropriate $[13, \underline{14}, \underline{21}$. But, we emphasize that we are dealing here with local operators.

With axioms 3 and $D^{\alpha} 1=0$, we show that the Mittag-Lefffler functions is an eigenfunction of this local metric derivative:

$D^{\alpha} E_{\alpha}\left(\lambda x^{\alpha}\right)=\lambda E_{\alpha}\left(\lambda x^{\alpha}\right)$

Of course, if we do not impose axiom 2 for Leibniz rule and substitute the axiom by another one that attributes the derivative of a constant to be zero, $D^{\alpha} 1=0$, the result above would be valid too. But, our intention is to permit the use o Leibniz rule, even if it would result in an approximation.

\section{From the Leibniz rule to the chain rule for local metric derivative}

We follow here a similar argument as the one presented in [13, 22], but restricting ourselves only to local derivatives and not fractional derivatives which are nonlocal and do not allow no-violated Leibniz rules.

Consider a deformed or metric derivative $D^{\alpha}$ of order $\alpha$ that satisfies the Leibniz rule (axiom 2), whose domain includes all locally Hölder-continuous functions of order $\alpha$. Using the Hadamard's representation theorem for $f \in C^{2}$, around an arbitrary point yields a fractional chain rule for such $D^{\alpha}$ applied to $f \circ w$, where $w$ is locally Hölder of exponent $\alpha$, accordingly to axiom 2 . Remember axiom 4 . Then the following statement holds:

$D_{x}^{\alpha}(f \circ w)\left(x_{0}\right)=f^{\prime}\left(w\left(x_{0}\right)\right)\left(D_{x}^{\alpha} w\right)\left(x_{0}\right)$.

This kind of chain rule holds for every kind of deformed derivatives [1, $\underline{18}, \underline{19}, \underline{23}, \underline{24}$.

\section{Comments: on local fractional derivative and the Jumarie's form of FC}

Despite the misunderstandings and missteps in the statements and formulations of certain authors [9, 10, 11, 24], it is worthy to highlight here some comments on possible limits of validity and looking for a new mathematics. Given that the Jumarie's formalism is based on the construction of a variant of FC theory, this formalism could be seen as an approximation (in that the Mittag-Leffler function is an eigenfunction of the fractional derivative operator proposed, keeping certain similarities with Caputo formalism of FC, yet, considering a low-level of fracionality limit). That is, the Leibniz rule or the chain rule could apply to non-local fractional operators, what actually happens not to be valid, unless we take the low-limit fracionality[14, 13, 21]. However, in a recent article [1], we have shown that the Jumarie's formalism seems to be actually related to a local deformed derivative operator and it is not really a fractional calculus formalism. Thus, we can consider it as an approximation, valid for low-fracionality limit, as shown in this contribution by means of the axiomatic form.

Regarding the formalism called local fractional derivative [24], this controversial local version is nothing but the adaptation of Jumarie's formalism, and therefore is only valid for low fracionality; moreover, it carries in itself certain misconceptions of Jumarie's formalism.

Also, taking into consideration the view of the author in Refs. $[13, \underline{14}, \underline{21}$ to deny alternatives to the usual FC formalism, our view here is based on the attempt to look for a new mathematics that could be better suited to the description of physical phenomena and can better model such phenomena, especially in dealing within the context of complexity.

\section{CONCLUSIONS AND OUTLOOK}

In short: we have derived an axiomatic version of metric derivative that has the Mittag-Leffler function as an eigenfunction and is only valid for low-level fractionality systems.

The structure of the deformed derivative generated shows similarities with some versions claimed to hold or not hold in the literature, but here it is axiomatically set up and the consistency are given in the context of low level of fractionality.

We obtain a chain rule for a metric derivative of order $\alpha \cong 1$ whose domain includes $C^{1}$ or even locally Hölder-continuous functions (which describe coarse-grained media), provided the Leibniz rule holds.

On the applicability of our approach proposed here, some comments are deserved. Certain systems in Nature seem to be well-described by some fractional parameter in a low-level limit of fractionality. Examples of such low-level systems are the 
anomalous magnetic g-factor of the charged leptons of the Standard Model, where the low level of fractionality seems to be consistent with the Quantum Electrodynamics corrections [25]. The possibility of constraining fractal space dimensionality from astrophysics and other areas [26], also points to a fractal dimension very close to an integer value, but still noninteger. Thus, the importance of this formalism that it may indicate some higher-order effects that might exist in the systems under study, without the cumbersome calculations of QED.

Other interesting applications are referred in the result of works from, for example, Ostoja et al., see e.g. Refs.[27, 28] with continuum models on fractal porous domains. These works use the Jumarie's definition of derivatives and are highly cited, in a positive way, but one of the main criticisms of the approach, as it can be seen, for example, in Refs. [29, 30], indicates that the approach into consideration, makes use of the MRL derivative and does have applicability. But here we have shown that it is an approach valid within a low-fractionality limit and it is in essence a local formalism.

\section{ACKNOWLEDGMENTS}

The authors wish to express their gratitude to FAPERJ-Rio de Janeiro and CNPq-Brazil for the partial financial support.

\section{REFERENCES}

1. J. Weberszpil, Matheus Jatkoske Lazo and J.A. Helayël-Neto, Physica A 436, (2015) 399-404.

2. Weberszpil, J.; Helayël-Neto, J.A., Physica. A (Print), v. 450, (2016) 217-227; arXiv:1511.02835 [math-ph].

3. C. Tsallis, J. Stat. Phys. 52, (1988) 479-487.

4. C. Tsallis, Brazilian Journal of Physics, 39, 2A, (2009) 337-356.

5. C. Tsallis, Introduction to Nonextensive Statistical Mechanics - Approaching a Complex World (Springer, New York, 2009).

6. Alexander S. Balankin and Benjamin Espinoza Elizarraraz, Phys. Rev. E 85, (2012) 056314.

7. A. S. Balankin and B. Espinoza, Phys. Rev. E 85, (2012) 025302(R).

8. Alexander Balankin, Juan Bory-Reyes and Michael Shapiro, Phys A, in press, (2015) doi:10.1016/j.physa.2015.10.035.

9. Jumarie, G., Math. Comput. Appl. (2006), pp 13671376.

10. Jumarie, G., Appl. Math. Letters (2009) Vol 22, pp 378382.

11. Jumarie G. Open Phys (2013) 11(6):617-33.

12. W. Chen, Chaos, Solitons and Fractals 28 (2006) 923-929.

13. Vasily E. Tarasov, Commun Nonlinear Sci Numer Simulat 18 (2013) 2945-2948.

14. Vasily E. Tarasov, Commun NonlinearSciNumerSimulat30(2016)1-4.

15. Jigen Peng, Kexue Li, J. Math. Anal. Appl. 370 (2010) 635-638.

16. Cheng-shi Liu, Commun Nonlinear Sci Numer Simulat 22 (2015) 92-94.

17. Manuel D. Ortigueira, J. A. Tenreiro Machado, Journal of Computational Physics 293 (2015) 4-13.

18. Khalil, R., Al Horani, M., Yousef. A. and Sababheh, M., J. Comput. Appl. Math. 264, (2014) 6570.

19. Douglas R. Anderson and Darin J. Ulness, JOURNAL OF MATHEMATICAL PHYSICS 56, (2015) 063502.

20. Ernesto P. Borges, Physica A 340 (2004) 95-101.

21. Vasily E. Tarasov, Fractals, Vol. 23, No. 2 (2015) 1575001.

22. J. Weberszpil, Proceeding Series of the Brazilian Society of Applied and Computational Mathematics, 3,N. 1 (2015) 010219-1-010219-5.

23. Yan Chen, Ying Yan, Kewei Zhang, J. Math. Anal. Appl. 362 (2010) 17-33.

24. Xiao-Jun Yang, H.M. Srivastava, Ji-Huan He, Dumitru Baleanu, Physics Letters A 377 (2013) 1696-1700.

25. Weberszpil, J.; Helayël-Neto, J. A., Advances in High Energy Physics, (2014), p. 1-12.

26. F. Caruso and V. Oguri, The Astrophysical Journal, 694 (2009)151-153.

27. J. Li, M. Ostoja-Starzewski, Proc. R. Soc. A 465 (2108) (2009) 2521-2536; J. Li, M. Ostoja-Starzewski, Proc. R. Soc. A 4672128 (2011) 1214.

28. P.N. Demmie, M. Ostoja-Starzewski, J. Elasticity 104 (1-2) (2011) 187-204.

29. Vasily E. Tarasov, Wave Motion 63 (2016) 18-22.

30. Vasily E. Tarasov, Journal of Math. Physics 55, (2014) 083510. 